\title{
A simulation study for one-pion exchange contribution on very forward neutron productions in ATLAS-LHCf common events
}

\author{
Ken Ohashi, ${ }^{a, *}$ Hiroaki Menjo, ${ }^{a}$ Yoshitaka Itow ${ }^{a, b}$ and Takashi Sako ${ }^{c}$ \\ ${ }^{a}$ Institute of Space-Earth Environmental Research, Nagoya University \\ Furo-cho, Chikusa-ku, Nagoya, 464-8601, Japan \\ ${ }^{b}$ Kobayashi-Maskawa Institute of the Origin of Particles and the Universe, Nagoya University \\ Furo-cho, Chikusa-ku, Nagoya, 464-8601, Japan \\ ${ }^{c}$ Institute for Cosmic Ray Research, the University of Tokyo \\ 5-1-5 Kashiwa-no-Ha, Kashiwa, Chiba, 277-8582, Japan \\ E-mail: ohashi.ken@isee.nagoya-u.ac.jp
}

Interpretations of the mass composition of ultra-high energy cosmic rays are unclear owing to uncertainties in the hadronic interaction models. Therefore, validations of hadronic interaction models via experiments are required. Recently, the LHCf experiment demonstrated differences in very forward neutron productions between data and predictions using hadronic interaction models for pseudo-rapidity $\eta>10.76$. Contributions of the one-pion exchange process, diffractive collisions, and non-diffractive collisions are possible sources of these differences. These three contributions exhibit different effects on the mass-sensitive observables of ultra-high energy cosmic-ray experiments; hence, it is crucial to measure these contributions separately. In this study, we present a method for separating the contribution of the one-pion exchange process from contributions of diffractive and non-diffractive collisions in ATLAS and LHCf detectors, considering detector resolutions in LHC-Run2 and Run3.

$37^{\text {th }}$ International Cosmic Ray Conference (ICRC 2021)

July 12th - 23rd, 2021

Online - Berlin, Germany

\footnotetext{
${ }^{*}$ Presenter
} 


\section{Introduction}

The mass composition of ultra-high energy cosmic rays is a piece of key information required to understand their origin. Mass-sensitive observables, such as depth of the maximum of shower developments $\left\langle X_{\max }\right\rangle$, depth of the maximum of muon productions $\left\langle X_{\max }^{\mu}\right\rangle$, and number of muons on the ground $N_{\mu}$, are measured in ultra-high energy cosmic-ray experiments, and mass composition is interpreted by comparing experimental results with simulations. However, there are large uncertainties in interpretations owing to the hadronic interaction models adopted in simulations, and these uncertainties are larger than those owing to systematic uncertainty in experiments. Therefore, for reliable interpretations, it is necessary to validate and improve hadronic interaction models using accelerator experiments.

The LHCf experiment [1] is an experiment that measures very forward neutral particles using the Large Hadron Collider for the validation of hadronic interaction models. Recently, they published production cross-sections of very forward neutrons [2], and reported large differences between data and predictions by hadronic interaction models for pseudo-rapidity $\eta>10.76$. Contributions of diffractive collisions and the one-pion exchange process are possible sources of these differences. The cross-section of diffractive collisions exhibit large effects on both $\left\langle X_{\max }\right\rangle$ and $\left\langle X_{\max }^{\mu}\right\rangle$ predictions [3]. However, measurements of the one-pion exchange process involve connecting pion-proton collisions, and the understanding of pion-proton collisions is more important for predictions of $\left\langle X_{\max }^{\mu}\right\rangle$ than for $\left\langle X_{\max }\right\rangle$ [4]. The other contribution, non-diffractive collisions, is also a possible source of these differences. Because these possible sources exhibit different effects on the predictions of mass-sensitive observables, it is important to understand what creates these differences by experiments.

A method for measuring the contributions of the one-pion exchange process, assuming the experimental setup by the CMS experiment, is discussed in Ref. [5]. Moreover, the contributions of the one-pion exchange process and total pion-proton cross-section are obtained from experimental data via the LHCf experiment [8] in Ref.[6, 7]. In these studies, they rely on one hadronic interaction model or theoretical calculations to estimate contributions from diffractive and nondiffractive collisions, as well as reduce or subtract these contributions. In the method presented in Ref. [5], the researchers only consider PYTHIA 6 for the estimations. In Ref. [6, 7], the researchers carried out estimations via theoretical calculations. However, as discussed in [9], there are very large uncertainties in these contributions, and these uncertainties are not considered in these studies; PYTHIA 8 [10,11] with DL tune[12] and EPOS-LHC [13] exhibit large diffractive contributions and small non-diffractive contributions on very forward neutron spectrum, while QGSJET II-04 [14] and SIBYLL $2.3[15,16]$ exhibit large non-diffractive contributions and small diffractive contributions. To understand the source of differences between results by the LHCf experiment and predictions, despite large uncertainties in predictions of diffractive and non-diffractive contributions, we need to separate the contributions of diffractive collisions, non-diffractive collisions, and the one-pion exchange process in the experiments. In this study, we develop a method for separating contributions of the one-pion exchange process from the other contributions using LHCf and ATLAS [17] detectors. The simulation setup and basic characteristics of one-pion exchange process are given in Section 2. ATLAS and LHCf detectors are introduced in Section 3. Then, the method is discussed in Section 4. We conclude the study in Section 5. 


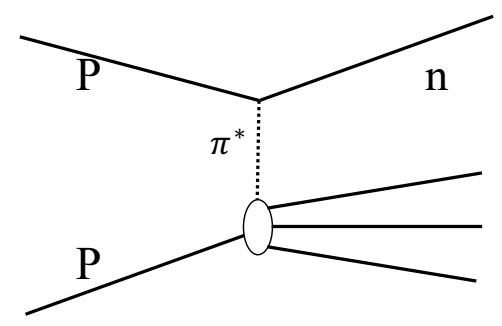

Figure 1: Feynman diagram of the one-pion exchange process.

\section{Simulation setup and characteristics of one-pion exchange process}

In this study, we adopt MonChER v1.0 [18] as the event generator of the one-pion exchange processes. To consider diffractive and non-diffractive collisions, EPOS-LHC, QGSJET II-04, and SIBYLL 2.3 in the interface CRMC v1.6 [19] are utilized. PYTHIA 8.212 with DL tune simulated by its interface is also adopted for background estimations. For the one-pion exchange, 500000 events are produced, while 100000000 events are produced for hadronic interaction models with $\sqrt{s}=13 \mathrm{TeV}$. The total inelastic cross-sections are $1.22 \mathrm{mb}$ and $0.088 \mathrm{mb}$ for the one-pion and $\rho$ and $a_{2}$ reggeon-exchange processes, respectively. In this study, we consider both the one-pion and $\rho$ and $a_{2}$ reggeon-exchange processes predicted by MonChER as signals. In addition, the diffractive and non-diffractive collisions predicted by hadronic interaction models are considered as backgrounds. In the results hereafter, we normalize the number of events by the total number of inelastic collisions. For the one-pion exchange simulated by MonChER, we normalize the number of events by $\sigma^{\mathrm{MonChER}} /\left(\sigma^{\text {inel }} \times N_{\text {col }}\right)$, where $\sigma^{\mathrm{MonChER}}=1.22+0.088 \mathrm{mb}$ and $\sigma^{\text {inel }}=79.5 \mathrm{mb}$ from the measurements by the TOTEM experiment[20]. $N_{c o l}$ represents the number of produced events by MonChER, which is 500000 .

The one-pion exchange process is the collision involving the exchange of a virtually charged pion. Figure. 1 presents the Feynman diagram of the one-pion exchange process. Particles are produced in the collision between a virtual pion and a proton, while a high energy neutron is produced in the very forward region. Transverse four-momentum loss of forward neutron, $t_{n}$, is defined as:

$$
t_{n}=\left(p_{\text {proton }}-p_{\text {neutron }}\right)^{2}=\left(p_{\pi^{+}}\right)^{2} \approx-\frac{p_{T}^{2}+\xi^{2} m_{p}^{2}}{1-\xi},
$$

where $p_{T}$ and $m_{p}$ represent neutron transverse momentum and is proton mass, respectively. In addition, $\xi$ depicts the neutron's relative energy loss defined as $\xi=\left(E_{p}-E_{n}\right) / E_{p}$, where $E_{p}$ and $E_{n}$ represent the energies of proton and neutron, respectively. Forward neutrons produced in this process are characterized by the high energy and low $t_{n}$, as illustrated in Figure. 2 and 3. The predictions obtained by the hadronic interaction models are also presented.

\section{ATLAS and LHCf detectors}

In this study, we consider two detectors: the LHCf-Arm2 detector and the ATLAS inner tracker. The LHCf detector is located $140 \mathrm{~m}$ away from the ATLAS interaction points. The LHCf-Arm2 detector comprises two sampling calorimeters with 1.4 interaction lengths made of 

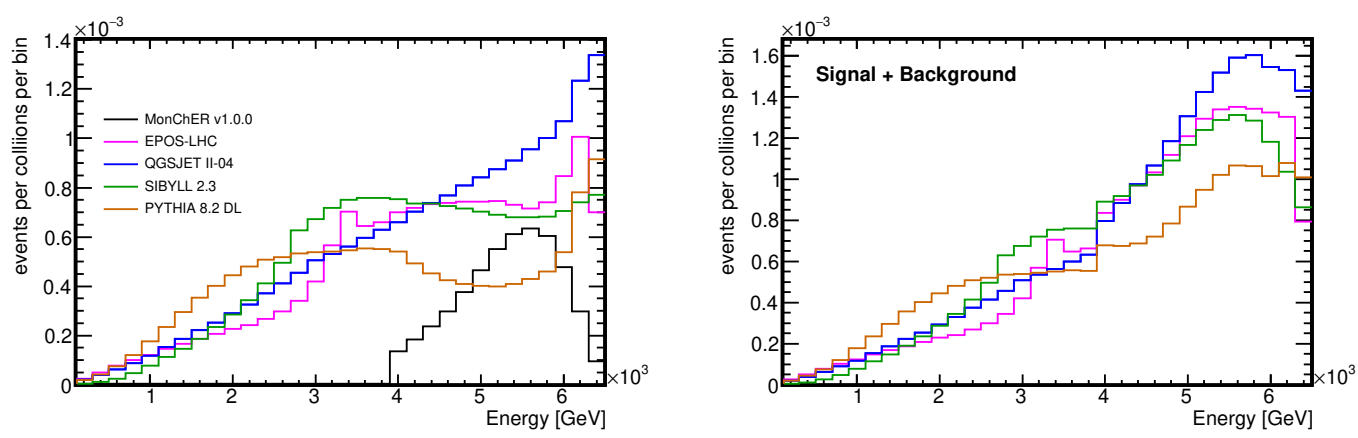

Figure 2: (left) True energy spectrum of neutrons in $\eta>10.76$ for the one-pion-exchange process (black), EPOS-LHC (magenta), QGSJET II-04 (blue), SIBYLL 2.3 (green), and PYTHIA 8.212 DL (orange). (right) True energy spectrum with one-pion exchange added to the predictions by four hadronic interaction models, considering one-pion exchange as signal and hadronic interaction models as backgrounds.

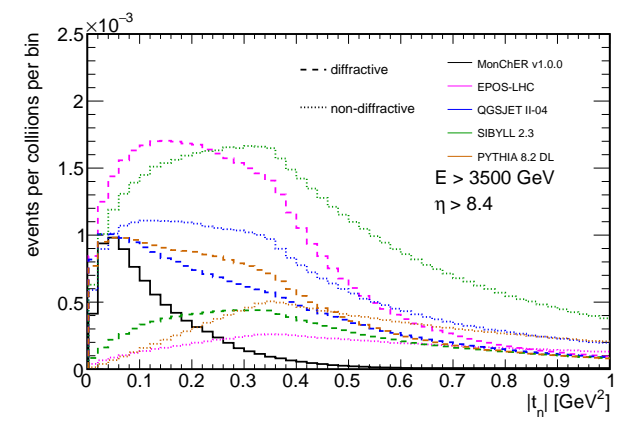

Figure 3: Neutron t distributions of neutrons with $E_{n}>3500 \mathrm{GeV}$ in $\eta>8.4$, corresponding to the pseudo-rapidity region covered by the LHCf detectors, for one-pion exchange (black), EPOS-LHC (magenta), QGSJET II-04 (blue), SIBYLL 2.3 (green), and PYTHIA 8.212 DL (orange). For hadronic interaction models, dotted lines depict the predictions for non-diffractive collisions, and dashed lines represent predictions for diffractive collisions.

tungsten plates and silicon strip sensors. The resolution of the LHCf detector for neutrons is 28$38 \%$ and $0.1-0.3 \mathrm{~mm}$ for energy and positions, respectively. The ATLAS inner tracker, which is an ATLAS detector, covers pseudo-rapidity from -2.5 to 2.5, and measuring charged particles with $p_{T}>100 \mathrm{MeV}$. Recently, ATLAS and LHCf collaborations demonstrate the results of the joint analysis of these detectors[21].

\section{Method for measuring one-pion exchange contributions on very forward neutron}

In the method for measuring the one-pion exchange contributions proposed in [5], the contributions of the diffractive collisions were minimized via the activity in the HF detector of the CMS experiment, and contributions of non-diffractive collisions were inhibited by requiring $\left|t_{n}\right|<0.2 \mathrm{GeV}^{2}$. To begin with, we consider a simple extension of the method for ATLAS and LHCf detectors without considering resolutions of the detector, and assess the effects of uncertainties owing to hadronic interaction models. As a simple extension, we require a neutron with $E_{n}>3500 \mathrm{GeV}$ in $\eta>10.76$, and $N_{c h}>10$, where $N_{c h}$ is the number of charged particles with $p_{T}>100 \mathrm{MeV}$ in 

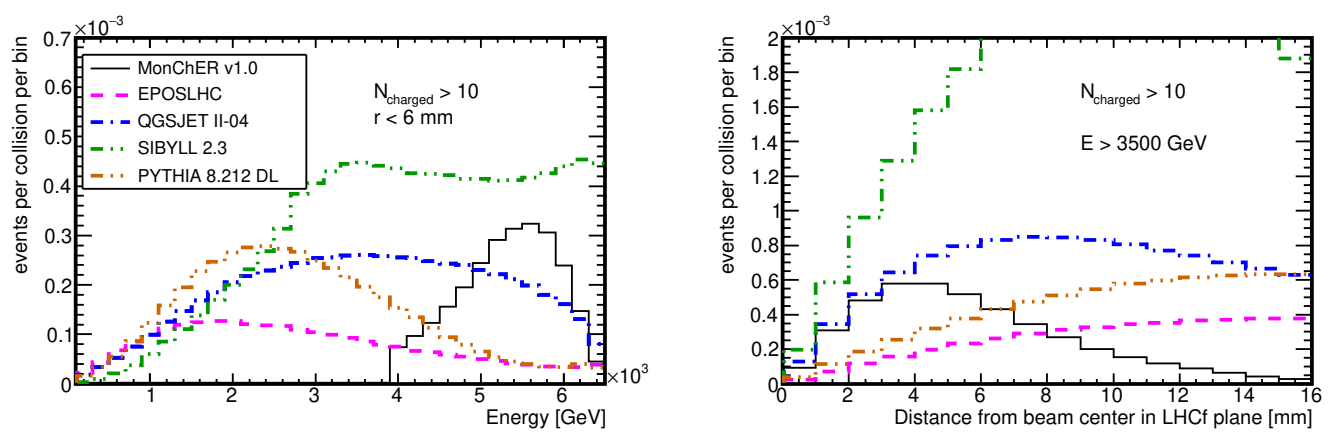

Figure 4: (left) True energy spectrum of neutrons for $N_{c h}>10$ in $\eta>10.76$ for one-pion exchange (black), EPOS-LHC (magenta), QGSJET II-04 (blue), SIBYLL 2.3 (green), and PYTHIA 8.212 DL (orange). (right) Hit distribution neutrons with distance from beam center in the LHCf plane for $N_{c h}>10$ and $E>3500 \mathrm{GeV}$.

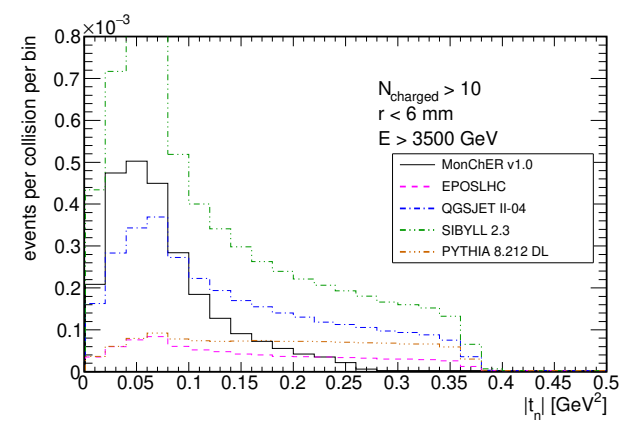

Figure 5: Neutron t distributions for $N_{c h}>10$ in $\eta>10.76$ for one-pion exchange (black), EPOS-LHC (magenta), QGSJET II-04 (blue), SIBYLL 2.3 (green), and PYTHIA 8.212 DL (orange).

the pseudo-rapidity region $|\eta|<2.5$, which is covered by the ATLAS inner tracker. As discussed in [9], most of events from diffractive collisions are $N_{c h}<5$; therefore, most of these diffractive contributions are rejected by selecting $N_{c h}>10$. By requiring a neutron with $E_{n}>3500 \mathrm{GeV}$ hit in $\eta>10.76$, neutrons with $\left|t_{n}\right|>0.4 \mathrm{GeV}^{2}$ are rejected. Fig. 4 illustrates the neutron energy spectra and hit distribution with a distance from the projected beam center on the LHCf detector surface, $r$, which are simulated by MonChER (primarily one-pion exchange process, signals) and hadronic interaction models (backgrounds). Note that $\eta>10.76$ corresponds to the region within $r<6 \mathrm{~mm}$ on the LHCf detector surface. There are clear differences in the shape of distributions for both the energy spectra and neutron hit distributions between signals and backgrounds, while very large backgrounds are estimated by QGSJET II-04 and SIBYLL 2.3. Fig. 5 presents the $\left|t_{n}\right|$ distributions after this event selection. The method in Ref. [5] and its simple extension successfully reduces backgrounds for PYTHIA 8.212 DL and EPOS-LHC cases because they predict small non-diffractive backgrounds for the low $\left|t_{n}\right|$ region. However, large non-diffractive backgrounds are estimated by QGSJET II-04 and SIBYLL 2.3, which cannot be reduced by this criteria. For the separation of signals from non-diffractive backgrounds, despite large uncertainties in background estimations, we need additional criteria.

To separate the contributions of the one-pion exchange process and non-diffractive backgrounds, the additional criteria, $N_{c h}>60$, is introduced. With large central activities such as $N_{c h}>60$, the 

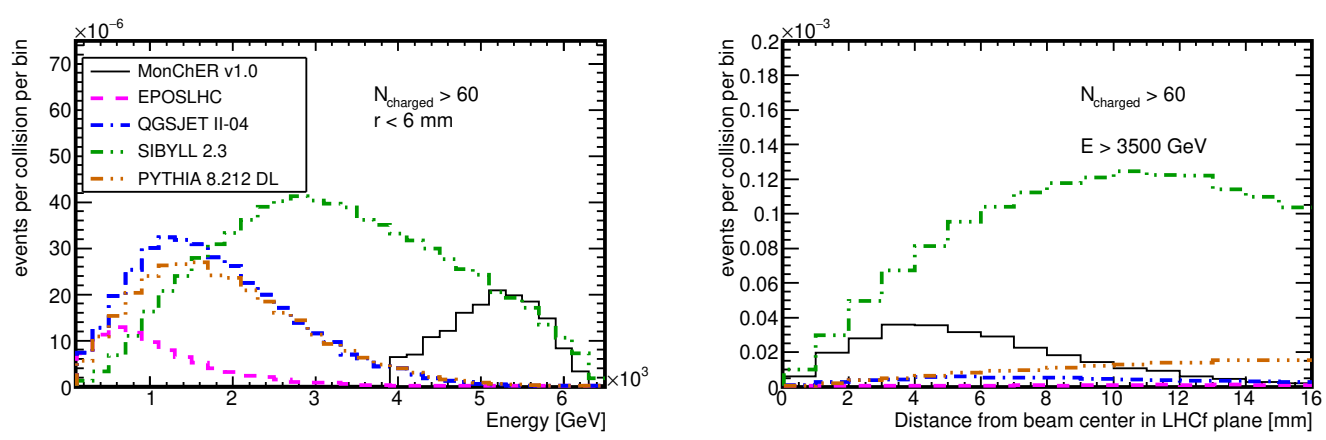

Figure 6: (left) True energy spectrum of neutrons for $N_{c h}>60$ in $\eta>10.76$ for the one-pion exchange (black), EPOS-LHC (magenta), QGSJET II-04 (blue), SIBYLL 2.3 (green), and PYTHIA 8.212 DL (orange). (right) Neutron hit distributions with distance from beam center in the LHCf plane for $N_{c h}>60$ in $\eta>10.76$.

number of multi-parton interactions is large, and this reduces the forward neutron energy for nondiffractive backgrounds. However, for the one-pion exchange processes, the number of multi-parton interactions between a virtual pion and a proton is large; however, this has a negligible effect on the forward neutron energy. Figure. 6 presents the energy spectrum for this case. If we consider spectra with one-pion-exchange signals and non-diffractive backgrounds, we can determine the two-peak structure with a signal peak and a background peak, and can separate the contributions into the signal sample and the non-diffractive background sample, except for the case with SIBYLL 2.3. Once we can separate them into two samples using the two-peak structure, we can constrain and tune predictions of non-diffractive collisions in hadronic interaction models using a background sample, as well as measure the cross-section and multiplicity distributions ( $N_{c h}$ distributions) for $N_{c h}>10$, where diffractive contributions are negligible, by subtracting non-diffractive contributions. If the contributions of non-diffractive backgrounds or one-pion exchange signals are significantly larger than the other contribution, we can obtain a one-peak structure in the energy spectrum as SIBYLL 2.3. In that case, we cannot separate the contributions. However, we can discuss the upper limit of the contribution of the one-pion exchange process because there are significant differences in the neutron energy spectrum and the neutron hit distribution between signals and non-diffractive backgrounds, as presented in Fig. 6.

In previous discussions, the resolutions of detectors were not considered. The resolution of the LHCf detector for neutrons is $38 \%$ at high energy. In LHC-RUN 3, the joint operation between LHCf and ATLAS ZDC is planned, and in this case, the energy resolution for neutrons is expected to be $20 \%$ [22]. To consider these resolutions, energy and positions are smeared using Gaussian distributions. For each neutron, $\mathrm{x}$ and y positions at the LHCf-Arm 2 detector surface are calculated, and Gaussian smearing, with a $0.3-\mathrm{mm}$ standard deviation, is applied for both $\mathrm{x}$ and y positions. The energy of neutrons is smeared with the Gaussian distributions with $40 \%$ and $20 \%$ standard deviations. Left and right plots of Figure. 7 present energy spectra with $40 \%$ and $20 \%$ Gaussian smearing for neutron energy with position smearing, respectively. With $40 \%$, it is difficult to determine a two-peak structure in the energy spectrum even if there are clear differences in true level. However, with $20 \%$ Gaussian smearing, there is a possibility of determining a two-peak structure. This result indicates the possibility of separating contributions of the one-pion exchange 

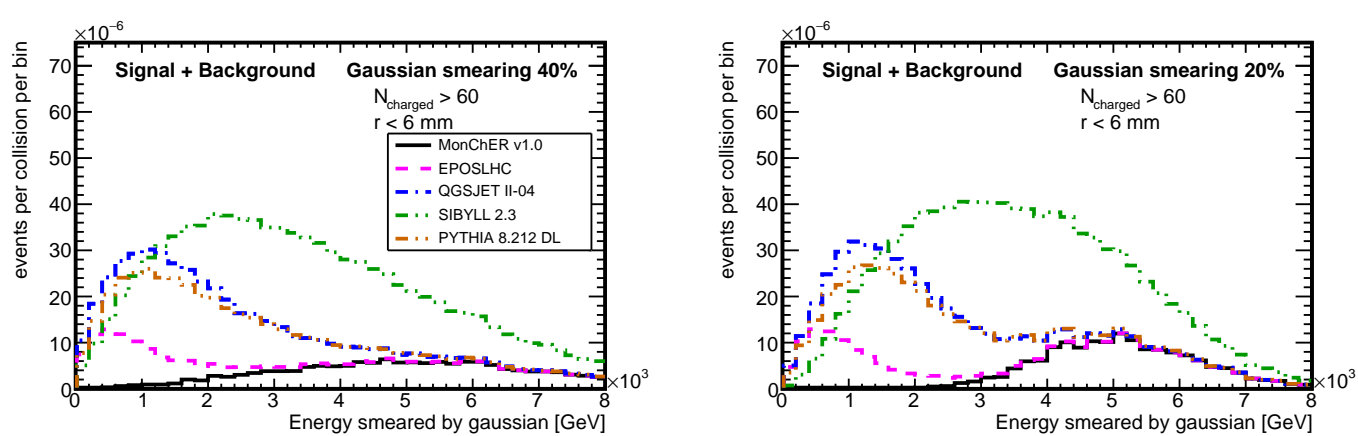

Figure 7: (left) Energy spectrum of neutrons after 40\% gaussian smearing with the prediction for one-pion exchange process added to the predictions by four hadronic interaction models, considering the one-pion exchange as signals and hadronic interaction models as backgrounds for EPOS-LHC (magenta), QGSJET II04 (blue), SIBYLL 2.3 (green), and PYTHIA 8.212 DL (orange). The prediction for the one-pion exchange process is also depicted by the black line. Events with $N_{c h}>60$ and neutrons with $E>3500 \mathrm{GeV}$ in $\eta>10.76$ are selected. (right) Energy spectrum of neutrons after $20 \%$ gaussian smearing.

signals and non-diffractive backgrounds in the event-by-event bias in LHC-RUN3.

\section{Conclusion}

In this study, we developed a method for separating the one-pion exchange contribution on very forward neutron productions from other contributions using ATLAS and LHCf detectors. The simple extension of the method discussed in Ref. [5] did not work optimally owing to uncertainties in the predictions of non-diffractive collisions; the significant contributions of non-diffractive collisions were predicted by some of the hadronic interaction models, which were not considered in Ref. [5]. By selecting events using $N_{c h}>60$, we were able to determine a two-peak structure with signal and background peaks in the neutron energy spectrum, except for the case with very large non-diffractive contributions, better than the one-pion exchange contribution. With the twopeak structure, we could clearly separate contributions of the one-pion exchange process and non-diffractive backgrounds into two samples. This facilitates the selection of the best model for background (non-diffractive contributions) predictions or tuning the prediction using the sample, which enables us to measure the cross-sections and multiplicity distributions of the one-pion exchange process for $N_{c h}>10$. For the case without a two-peak structure, we could not separate the contributions, although we could discuss the upper limit of the contribution of the one-pion exchange process using the neutron energy spectrum and the neutron hit distribution on the LHCf surface. Resolutions of detectors were also considered. The energy resolution of the current LHCf detector was insufficient in determining the two-peak structure and applying the method presented in this study. In LHC-RUN3, the joint operation between LHCf and ATLAS ZDC detectors was planned, and energy resolutions for the neutrons were expected to be $20 \%$. In this case, it is possible to apply the method discussed in this study.

\section{Acknowledgements}

This research was supported by JSPS KAKENHI Grant Number JP21J11122. 


\section{References}

[1] LHCf Collaboration, CERN-LHCC-2006-004 (2006). https://cds.cern.ch/record/926196.

[2] O. Adriani, E. Berti, L. Bonechi et al., JHEP11(2018)073

[3] K. Ohashi, H. Menjo, Y. Itow, T. Sako, K. Kasahara, Progress of Theoretical and Experimental Physics, Volume 2021, Issue 3, March 2021, 033F01, https://doi.org/10.1093/ptep/ptab013

[4] T. Pierog, EPJ Web of Conferences 208, 02002 (2019)

[5] V.A. Petrov et. al, Eur. Phys. J. C (2010) 65: 637 - 647

[6] R. A. Ryutin, Eur. Phys. J. C (2017) 77:114

[7] V. A. Khoze, A. D. Martin, and M. G. Ryskin, Phys. Rev. D 96, 034018 (2017)

[8] O. Adriani et al. (LHCf Collaboration), Phys. Lett. B 750, 360 (2015)

[9] Qi-Dong Zhou et al, Eur. Phys. J. C (2017) 77:212

[10] T. Sjostrand, S. Mrenna, and P. Z. Skands, JHEP05(2006) 026, arXiv:hep-ph/0603175 [hep$\mathrm{ph}]$.

[11] T. Sjostrand, S. Mrenna, and P. Z. Skands, Comput. Phys. Commun.178(2008) 852-867,arXiv:0710.3820 [hep-ph]

[12] A. Donnachie and P. V. Landshoff, Nucl. Phys. B244(1984) 322.

[13] T. Pierog, I. Karpenko, J. M. Katzy, E. Yatsenko, and K. Werner, Physical Review C 92 (2015) 034906.

[14] S. Ostapchenko, Physical Review D 83 (2011) 014018.

[15] E.-J. Ahn, R. Engel, T. K. Gaisser, P. Lipari, and T. Stanev, Physical Review D 80 (2009) 094003.

[16] F. Riehn et al., PoS ICRC2017 (2017) 301.

[17] ATLAS Collaboration, JINST 3 (2008) S08003.

[18] R.A. Ryutin, A.E. Sobol, and V.A. Petrov, arXiv:1106.2076; R.A. Ryutin, A.E. Sobol, and V.A. Petrov, https://moncher.hepforge.org/

[19] T. Pierog, C. Baus, R. Ulrich, https://web.ikp.kit.edu/rulrich/crmc.html

[20] G. Antchev et al., Eur. Phys. J. C (2019) 79:103

[21] ATLAS and LHCf Collaborations, Technical Report ATLAS-CONF-2017-075 (CERN, Geneva, 2017).

[22] LHCf Collaboration, LHCf - Technical Proposal for the LHC Run3, CERN-LHCC-P-014 (CERN, Geneva, 2019) 